\title{
Self-Induced Resonances in Asymmetric Superconducting Quantum Interference Devices
}

\author{
T.P. POLAK \\ Faculty of Physics, Adam Mickiewicz University of Poznań \\ Umultowska 85, 61-614 Poznań, Poland \\ AND E. SARNELLI \\ Consiglio Nazionale delle Ricerche - \\ Istituto Nazionale per la Fisica della Materia \\ Complesso Universitario Monte S. Angelo, 80126 Naples, Italy \\ Theory of self-induced resonances in asymmetric two-junction interfer- \\ ometer device is presented. An extension of previous theoretical approaches \\ contains inclusions from several asymmetries: the Josephson current $\epsilon$, ca- \\ pacitances $\chi$ and dissipation $\rho$ presented in an equivalent circuit. Our theory \\ can be useful to determine asymmetry parameters always present in lightly \\ damped asymmetric SQUIDs made from low- and high- $T_{\mathrm{C}}$ materials.
}

PACS numbers: 74.72.- $\mathrm{h}, 74.50 .+\mathrm{r}$

\section{Introduction}

Superconducting quantum interference devices (SQUIDs) are the most employed superconductive electronic circuits in practical applications [1]. With the discovery of high-temperature superconductors (HTS) also high-temperature SQUIDs have been developed [2]. This class of devices, although less sensitive than the most competitive low-temperature SQUIDs, have been used in several applications, where portability and/or positioning as much as high working temperatures are needed. Moreover, the demonstration of an unconventional symmetry of the order parameter in $\mathrm{YBaCuO}$ (YBCO) [3] opened new horizons for using the so-called pi-SQUIDs in superconductive electronics. As a consequence, a full knowledge of properties of HTS SQUIDs is at great importance.

The outline of the paper is the following: firstly, we outline the model Hamiltonian, and present equations for asymmetric dc-SQUIDs. Next, we consider the method and assumptions which have been made. Finally we discuss obtained results and their relevance to the experimental situations. 


\section{Model}

We start with defining an asymmetric superconducting quantum interference device (ASQUID) which consists of two Josephson junctions. Each of them has a critical current $I_{\mathrm{C} i}$ and a parallel capacitance $C_{i}$. We assume also that single junction contains a parallel linear resistance $R_{i}$ and interferometer is fed by an external source $I_{\mathrm{c}}$. The self-inductances of the junctions in ASQUID are equal to $L_{1}$ and $L_{2}$ and Hamiltonian contains three parts [4]:

$$
\mathcal{H}=\mathcal{H}_{\mathrm{C}}+\mathcal{H}_{\mathrm{J}}+\mathcal{H}_{\mathrm{M}}
$$

First term on the right side of Eq. (1) defines electrostatic energy

$$
\mathcal{H}_{\mathrm{C}}=\frac{1}{2}\left(\frac{\Phi_{0}}{2 \pi}\right)^{2}\left(C_{1} \dot{\phi}_{1}^{2}+C_{2} \dot{\phi}_{2}^{2}\right),
$$

where $\phi_{i}$ is a phase difference across the $i$-th junction and $\Phi_{0}=h c / 2 e$ is the flux quantum. The second term is the Josephson energy

$$
\mathcal{H}_{\mathrm{J}}=E_{\mathrm{J}, 1}\left(1-\cos \phi_{1}\right)+\mathrm{E}_{\mathrm{J}, 2}\left(1-\cos \phi_{2}\right),
$$

where $E_{\mathrm{J}, i}=\left(\Phi_{0} / 2 \pi\right) I_{\mathrm{C}, i}$. To complete the set of equations for the interferometer one should take into account that loop current $I_{\mathrm{L}}$ can contribute to the flux and as a consequence magnetic energy takes the form

$$
\mathcal{H}_{\mathrm{M}}=\frac{1}{2}\left(\frac{\Phi_{0}}{2 \pi}\right)^{2} \frac{\left(\phi_{2}-\phi_{1}-\phi_{\text {ext }}\right)^{2}}{L_{+}},
$$

where $L_{+}=L_{1}+L_{2}$. We introduce the following parameters: $C_{1,2}=(1 \pm \chi) C$, $E_{J, 1,2}=(1 \pm \epsilon) E_{\mathrm{J}}, L_{1,2}=(1 \pm \lambda) L / 2$, where dimensionless anisotropy quantities $\chi, \epsilon$, and $\lambda$ describe the relative deviations of the model parameters from the corresponding average values $C, E_{\mathrm{J}}$ and $L$. Considering dissipation due to a quasiparticle current we add parallel resistances $R_{i} \sim \alpha_{i}^{-1}$. We assume that SQUID is current excited by a constant current source. This foundation leads to an additional term $\gamma_{i}$ in both equations. Finally, we write the equations for ASQUID, after applying the Euler-Lagrange equation to the Lagrangian and renormalization, in dimensionless form

$$
\begin{aligned}
& (1+\chi) \ddot{\phi}_{1}+(1+\rho) \alpha \dot{\phi}_{1}+(1+\epsilon) \sin \left(\phi_{1}\right)=\gamma_{1}+\frac{\phi_{2}-\phi_{1}}{\beta}, \\
& (1-\chi) \ddot{\phi}_{2}+(1-\rho) \alpha \dot{\phi}_{2}+(1-\epsilon) \sin \left(\phi_{2}\right)=\gamma_{2}+\frac{\phi_{1}-\phi_{2}}{\beta} .
\end{aligned}
$$

Choice of parameters $\chi=\epsilon=\rho=0$ stands for the fully symmetric case.

\section{Results}

We shall analyze two coupled differential Eqs. (5) and (6) for the case $\beta \leq 1$ that coupling between the two junctions of the interferometer is strong. We assume voltage sinusoidal variations with dc component $V$, ac amplitude $v$, frequency $\omega$ and phase $\varphi: V(t)=V+v \cos (\omega t+\varphi)$. Extracting from Eqs. (5) and (6) the dc, $\sin \omega t$ and $\cos \omega t$ Fourier components we get 

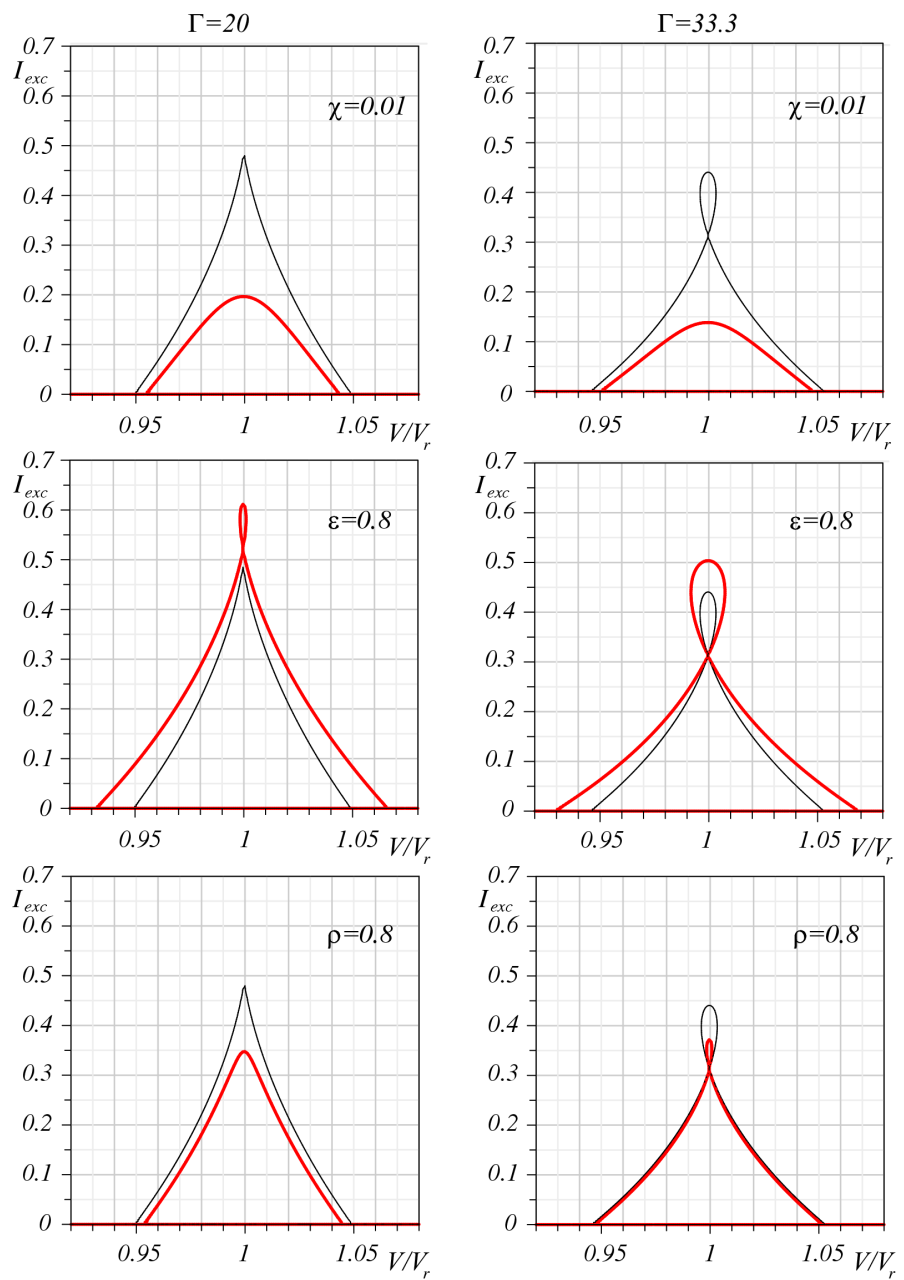

Fig. 1. The normalized resonant current $I_{\text {exc }}$ versus normalized voltage $V / V_{\mathrm{r}}$, with different values of the anisotropy parameters, capacitance $\chi$, the Josephson current $\varepsilon$, and dissipation $\rho$ for second resonance $(n=2)$. Black curves refer to absence of anisotropy parameters.

$$
I_{\text {exc }}=\frac{\alpha \delta^{2} \omega}{2 n},
$$

where $\tilde{\omega}=\omega / \omega_{\mathrm{r}}=V / V_{\mathrm{r}}$ is the normalized voltage and $\omega_{r}$ is the resonant frequency.

$$
\left[\frac{\delta\left(1-\tilde{\omega}^{2}\right)}{J_{n}^{-}(\delta)}\right]^{2}+\left[\frac{\alpha \delta \tilde{\omega}}{J_{n}^{+}(\delta)}\right]^{2}+\left[\frac{\chi \delta \tilde{\omega}^{2}}{J_{n}^{-}(\delta)}\right]^{2}+\left[\frac{\alpha \rho \delta \tilde{\omega}}{J_{n}^{+}(\delta)}\right]^{2}=1+\epsilon^{2} .
$$

The influence of the dissipative current $\rho$ (see Fig. 1) manifests by the decrease in the maximum value of the resonant current, for given $n$-th resonance mode, 
when we increase the anisotropy parameter. However, even small deviations of the capacitative anisotropy parameter $\chi$ from equilibrium have a major impact on behavior of the ASQUID. For small values of $\chi$, at fixed value of the damping parameter $\Gamma \equiv\left(\alpha \omega_{\mathrm{r}}\right)^{-1}$ there are two possible solutions even for the first resonance. In symmetric SQUIDs this situation was present for higher resonances $n \geq 3[4]$.

\section{Discussion}

To produce the asymmetry of the Josephson current in the low- $T_{\mathrm{C}}$ interferometer we can change the area of the junction $A$ and in consequence the critical current can be written as $I_{\mathrm{C}}=j_{\mathrm{C}} A$, where $j_{\mathrm{C}}$ is the critical current density. However, from the Ambegaokar-Baratoff [5] formula we know that the product $I_{\mathrm{C}} R_{\mathrm{N}}$, where $R_{\mathrm{N}}$ is the resistance in normal state, has an invariant value which depends only on the material in fixed temperature. Thus changing the value of the Josephson current we alter the resistance of the junction $\left(\Delta I_{\mathrm{C}} \sim \Delta C \sim \Delta R^{-1}\right.$, see Fig. 2).
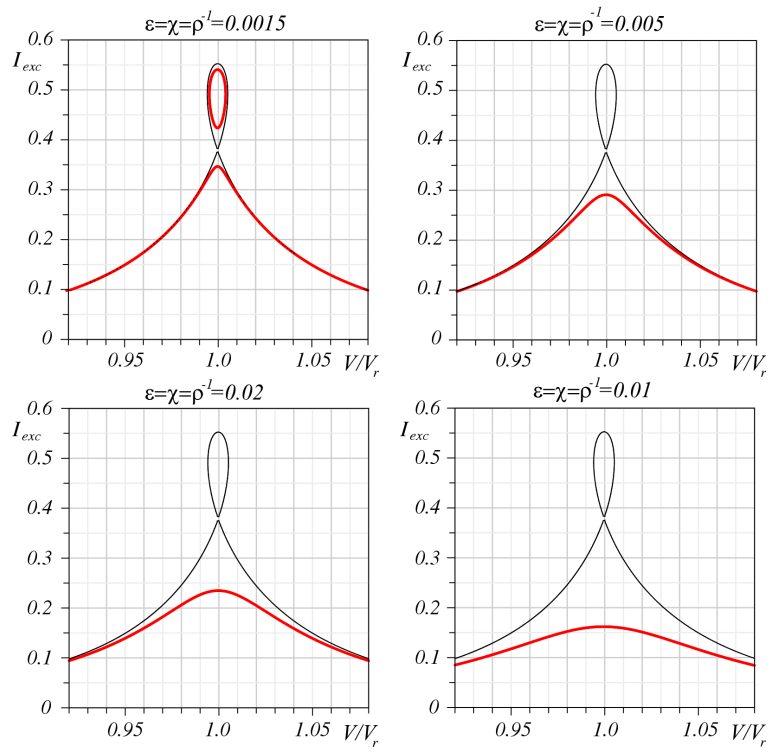

Fig. 2. Current voltage characteristics $\left(I_{\mathrm{exc}}-V / V_{\mathrm{r}}\right)$ for several asymmetric configurations of the SQUIDs related to the Ambegaokar-Baratoff formula, first resonance $n=1$, $\Gamma=20, \beta=0.1$.

For high- $T_{\mathrm{C}}$ SQUIDs the simple rule $I_{\mathrm{C}} R_{\mathrm{N}}=$ const valid for low- $T_{\mathrm{C}}$ SQUIDs does not apply in the case of interferometers based on the symmetric bicrystal $c$-axis [001] devices. In such ASQUIDs, $I_{\mathrm{C}} R_{\mathrm{N}}$ is proportional to the critical current density $J_{\mathrm{C}}$ at low values and stays roughly constant at high- $J_{\mathrm{C}}$ values and changing one single parameter is now possible [6]. 


\section{Summary}

In this paper we have presented an analytical approach that revealed the nature of the resonances in the presence of several asymmetries: the Josephson current $\epsilon$, capacitances $\chi$, and dissipation $\rho$. Our calculations imply that deviations of the capacitances from the average value in SQUID have profound impact on physics of the system. We have found that our theory can be useful to determine asymmetry parameters present in lightly damped ASQUIDs also produced from HTS materials.

\section{Acknowledgments}

Authors would like to thank Prof. Antonio Barone and Dr. Ciro Nappi for a lot of fruitful discussions. This work was supported by the TRN "DeQUACS".

\section{References}

[1] N. Bergeala, J. Lesueur, G. Faini, M. Aprili, J.P. Contour, Appl. Phys. Lett. 89, 112515 (2006).

[2] M. Kawasaki, P. Chaudhari, T.H. Newman, A. Gupta, Appl. Phys. Lett. 58, 2555 (1991).

[3] M. Sigrist, T.M. Rice, Rev. Mod. Phys. 67, 503 (1995).

[4] A. Barone, G. Paterno, Physics and Applications of the Josephson Effect, Wiley, New York 1982.

[5] V. Ambegeokar, A. Baratoff, Phys. Rev. Lett. 10, 486 (1963).

[6] E. Sarnelli, G. Testa, D. Crimaldi, A. Monaco, M.A. Navacerrada, Supercond. Sci. Technol. Rapid Commun. 18, L35 (1905). 